Pacific Journal of Mathematics

MIXING AUTOMORPHISMS OF COMPACT GROUPS AND A

KLAUS SCHмI 
Pacific Journal of Mathematics

MIXING AUTOMORPHISMS OF COMPACT GROUPS AND A

KLAUS SCHмI 


\title{
MIXING AUTOMORPHISMS OF COMPACT GROUPS AND A THEOREM BY KURT MAHLER
}

\author{
KLAUS SCHMIDT \\ Dedicated to the memory of Henry Abel Dye
}

\begin{abstract}
We investigate the higher order mixing properties of $\mathbb{Z}^{d}$-actions by automorphisms of a compact, abelian group and exhibit a connection between certain mixing conditions and a result by Kurt Mahler.
\end{abstract}

1. Introduction. Let $X$ be a compact, abelian group, and let $\operatorname{Aut}(X)$ denote the group of continuous automorphisms of $X$. We investigate the mixing behaviour of $\mathbb{Z}^{d}$-actions $\alpha: \mathbf{n} \rightarrow \alpha_{\mathbf{n}}$ on $X$ with the property that $\alpha_{\mathbf{n}} \in \operatorname{Aut}(X)$ for every $\mathbf{n} \in \mathbb{Z}^{d}$ (such an action will be called a $\mathbb{Z}^{d}$-action by automorphisms). If $(X, \alpha)$ satisfies the descending chain condition, i.e. if every decreasing sequence of closed, $\alpha$-invariant subgroups of $X$ eventually becomes constant, then $\alpha$ is algebraically and topologically conjugate to the shift action on a closed, shift invariant subgroup of $\left(\mathbb{T}^{k}\right)^{\mathbb{Z}^{d}}$, where $\mathbb{T}=\mathbb{R} / \mathbb{Z}$, and is automatically a Markov shift in $d$ dimensions (cf. [KS] for a more general result). Furthermore it is easy to see that the dual group $\hat{X}$ of $X$ can naturally be viewed as a finitely generated $R_{d}$-module, where $R_{d}$ is the ring of Laurent polynomials in $d$ variables with integral coefficients (cf. [KS]). In view of this correspondence between finitely generated $R_{d}$-modules and $\mathbb{Z}^{d}$ actions by automorphisms of compact, abelian groups the question arises how the algebraic properties of the $R_{d}$-module $M=\hat{X}$ reflect the dynamical properties of the $\mathbb{Z}^{d}$-action $\alpha$. In [S2] it was shown how to read off ergodicity, mixing, expansiveness, and certain facts about periodic orbits, from properties of the prime ideals associated with the $R_{d}$-module $M$. In this paper we continue this investigation and study the higher order mixing behaviour of such actions. This problem was raised by a paper of $F$. Ledrappier which contains examples of such actions which are (strongly) mixing, but which fail to be $r$-mixing for some $r \geq 2$. In these examples higher order mixing breaks down in a particularly interesting way: there exist a nonempty set $S \subset \mathbb{Z}^{d}$ and Borel sets $\left\{B_{\mathbf{n}} \subset X: \mathbf{n} \in S\right\}$ with positive Haar measure such that the sets $\left\{\alpha_{k \mathbf{n}}\left(B_{\mathbf{n}}\right): \mathbf{n} \in S\right\}$ fail to become asymptotically independent as $k \rightarrow \infty$. In order to simplify terminology we call the set $S$ a mixing 
shape if the sets $\left\{\alpha_{k \mathbf{n}}\left(B_{n}\right): \mathbf{n} \in S\right\}$ become asymptotically independent as $k \rightarrow \infty$, irrespective of the choice of $\left\{B_{\mathbf{n}} \subset X: \mathbf{n} \in S\right\}$.

If the action $\alpha$ is $r$-mixing for every $r \geq 1$, then every shape $S \subset \mathbb{Z}^{d}$ must obviously be mixing, but the converse is not at all clear. In $\S 3$ we prove the following results: if $\alpha$ is mixing and $X$ is connected, then every shape $S \subset \mathbb{Z}^{d}$ is mixing. If $X$ is zero dimensional, then there exist shapes which are not mixing, unless the prime ideals associated with the module $M=\hat{X}$ are all of the form $p_{i}=\pi_{i} R_{d}$, where $\pi_{i} \in \mathbb{Z}$ is a rational prime. Furthermore, if $X$ is zero dimensional, then $\alpha$ is $r$-mixing for every $r \geq 1$ if and only if every shape $S \subset \mathbb{Z}^{d}$ is mixing. I was unable to prove the corresponding result for connected groups.

The statement about mixing shapes for connected groups turns out to be intimately linked with a result due to Kurt Mahler [Ma]: if $c_{1}, \ldots, c_{m}$ and $a_{1}, \ldots, a_{m}$ are nonzero algebraic numbers, and if $c_{1} a_{1}^{k}+$ $\cdots+c_{m} a_{m}^{k}=0$ for infinitely many $k \geq 1$, then there exists an arithmetic progression $P \subset \mathbb{N}$ such that $c_{1} a_{1}^{k}+\cdots+c_{m} a_{m}^{k}=0$ for every $k \in P$. Since we need this result in a slightly strengthened form (with $c_{i}, a_{i}$ in an arbitrary field of characteristic 0) we include a proof of Mahler's result, adapted to this case, $\S 2$.

Finally a remark about notation: $\mathbb{C}, \mathbb{N}, \mathbb{Q}, \mathbb{R}$ will denote the complex, natural, rational and real numbers, and $\mathbb{T}=\mathbb{R} / \mathbb{Z}$. If $R$ is a ring then $R^{\times}$will stand for the group of units in $R$, and $R\left[x_{1}, \ldots, x_{n}\right]$ is the ring of polynomials in the variables $x_{1}, \ldots, x_{n}$ with coefficients in $R$. If $R=\mathbb{Z}$ or $\mathbb{Q}$, and if $I \subset R\left[x_{1}, \ldots, x_{n}\right]$ is an ideal, we set $V(I)=$ $\left\{\left(c_{1}, \ldots, c_{n}\right) \in\left(\mathbb{Q}^{-}\right)^{n}: f\left(c_{1}, \ldots, c_{n}\right)=0\right.$ for every $\left.f \in I\right\}$, where $\mathbb{Q}^{-}$is the algebraic closure of $\mathbb{Q}$.

Acknowledgment. I would like to thank the Institute for Advanced Study, Princeton, for hospitality and support during the Spring Term 1988, when this work was carried out. Special thanks are due to Enrico Bombieri for pointing out to me Mahler's paper [Ma] and for helpful discussions.

\section{Mahler's theorem.}

2.1. THEOREM([Ma]). Let $k$ be an algebraic number field of degree $(k: \mathbb{Q})=d, q \geq 2$, and let $\alpha_{1}, \ldots, \alpha_{q}$ be nonzero elements in $k$. Suppose that there exist an infinite sequence $m_{1}<m_{2}<\cdots$ in $\mathbb{N}$ and nonzero elements $c_{1}, \ldots, c_{q}$ in $k$ such that

$$
c_{1} \alpha_{1}^{m_{\jmath}}+c_{2} \alpha_{2}^{m_{\jmath}}+\cdots+c_{q} \alpha_{q}^{m_{\jmath}}=0
$$


for every $j \geq 1$. Let $p \geq 2$ be a rational prime and let $v$ be a valuation of $k$ above $p$ such that $\left|\alpha_{i}\right|_{v}=1$ for $i=1, \ldots, q$. Then there exist integers $a, b$ such that

$$
\begin{gathered}
1 \leq a<p^{2 d^{2}}, \quad 0 \leq b<a, \\
c_{1} \alpha_{1}^{j a+b}+c_{2} \alpha_{2}^{j a+b}+\cdots+c_{q} \alpha_{q}^{j a+b}=0 \quad \text { for every } j \geq 0,
\end{gathered}
$$

and

$$
\alpha_{i}^{a}=\alpha_{i^{\prime}}^{a} \text { for some } i, i^{\prime} \text { with } 1 \leq i<i^{\prime} \leq q .
$$

Proof. Denote by $k_{v}, I_{v}$, and $r_{v}$ the completion of $k$ with respect to $v$, the maximal compact subring of $k_{v}$, and the maximal ideal of $I_{v}$, respectively, and choose a prime element $\pi \in r_{v}$ (i.e. $|\pi|_{v}=$ $\sup \left\{|\alpha|_{v}: \alpha \in k\right.$ and $\left.\left.|\alpha|_{v}<1\right\}\right)$. Since $\operatorname{card}\left(k_{v} / r_{v}^{2 d}\right) \leq p^{2 d^{2}}$ we can find an integer $a$ with $1 \leq a<p^{2 d^{2}}$ such that $\alpha^{a} \equiv 1\left(\bmod r_{v}^{2 d}\right)$ for every $\alpha \in k$ with $|\alpha|_{v}=1$, and we set $\beta_{i} \pi^{2 d}=\alpha_{i}^{a}-1,1 \leq i \leq q$. Fix $i$ for the moment and observe that, for every $z \in \mathbb{N}$,

$$
\alpha_{i}^{a z}=\left(1+\beta_{i} \pi^{2 d}\right)^{z}=\sum_{j=0}^{\infty} \beta_{i}^{j} \pi^{2 d j}\left(\begin{array}{l}
z \\
j
\end{array}\right) .
$$

The polynomial

$$
P_{j}(z)=\left(\begin{array}{l}
z \\
j
\end{array}\right) \cdot j !=z \cdot(z-1) \cdot \cdots \cdot(z-j+1)
$$

has integral coefficients. For every $j \geq 0$, the highest power $t \geq 0$ such that $p^{t}$ divides $j$ ! satisfies that $t<j / p+j / p^{2}+\cdots=j /(p-1) \leq j$, and we conclude that $|j !|_{v}>\left|p^{j}\right|_{v} \geq\left|\pi^{d j}\right|_{v}$ and $\left|\pi^{d j} / j !\right|_{v}<1$. We can thus rearrange the sum in (2.6) as

$$
\sum_{j=0}^{\infty} \beta_{i}^{j} \pi^{2 d j} P_{j}(x) / j !=\sum_{j=0}^{\infty} \gamma_{i}(j) x^{j}=f_{i}(x),
$$

where $x \in I_{v}, \gamma_{i}(j) \in k_{v}$ and $\left|\gamma_{i}(j)\right|_{v} \leq\left|\pi^{d j}\right|_{v}$ for every $j \geq 0$, and obtain a well defined function $f_{i}: I_{v} \rightarrow I_{v}$ with $f_{i}(x)=\alpha_{i}^{a x}$ whenever $x \in \mathbb{N}$. Assume without loss in generality that $\left|c_{i}\right|_{v} \leq 1$ for $i=1, \ldots, q$, choose an integer $b$ with $0 \leq b<a$ and $m_{j} \equiv b(\bmod a)$ for infinitely many $j \geq 1$, and define $g: I_{v} \rightarrow I_{v}$ by

$$
\begin{aligned}
g(x) & =c_{1} \alpha_{1}^{b} f_{1}(x)+\cdots+c_{q} \alpha_{q}^{b} f_{q}(x) \\
& =\sum_{j=0}^{\infty}\left(c_{1} \alpha_{1}^{b} \gamma_{1}(j)+\cdots+c_{q} \alpha_{q}^{b} \gamma_{q}(j)\right) x^{j} .
\end{aligned}
$$


Note that $g$ is a power series which converges for every $x \in I_{v}$, and that there exists an infinite set $S \subset I_{v}$ such that $g(x)=0$ for every $x \in S$. Since $I_{v}$ is compact, $S$ has a limit point $y$, and $g(y)=0$. Exactly as in the case of a complex power series one concludes that $g(x)=0$ for every $x \in I_{v}$. In particular

$$
g(z)=c_{1} \alpha_{1}^{b} f_{1}(z)+\cdots+c_{q} \alpha_{q}^{b} f_{q}(z)=c_{1} \alpha_{1}^{a z+b}+\cdots+c_{q} \alpha_{q}^{a z+b}=0
$$

for every $z \in \mathbb{N}$, which proves (2.4). Condition (2.5) follows from the fact that

$$
\operatorname{det}\left(\begin{array}{ccc}
1 & \cdots & 1 \\
\alpha_{1}^{a} & \cdots & \alpha_{q}^{a} \\
\vdots & \ddots & \vdots \\
\alpha_{1}^{a(q-1)} & \cdots & \alpha_{q}^{a(q-1)}
\end{array}\right)=0
$$

2.2. Corollary. Let $m_{1}<m_{2}<\cdots$ be an infinite sequence in $\mathbb{N}$, and let $n \geq 2$. For every $1 \leq j_{1}<j_{2}<\cdots<j_{n}$ we define a polynomial

$$
P_{\left(j_{1}, \ldots, j_{n}\right)}\left(x_{1}, \ldots, x_{n}\right)=\operatorname{det}\left(\begin{array}{ccc}
x_{1}^{m_{J_{1}}} & \ldots & x_{n}^{m_{J_{1}}} \\
\vdots & \ddots & \vdots \\
x_{1}^{m_{J_{n}}} & \ldots & x_{n}^{m_{J_{n}}}
\end{array}\right)
$$

with integral coefficients, and we denote by $I \subset \mathbf{R}=\mathbb{Q}\left[x_{1}, \ldots, x_{n}\right]$ the ideal generated by $\left\{P_{\left(j_{1}, \ldots, j_{n}\right)}: 1 \leq j_{1}<\cdots<j_{n}\right\}$. Then there exists an integer $a \geq 1$ such that

$$
V(I) \subset V\left(\Delta\left(x_{1}^{a}, \ldots, x_{n}^{a}\right)\right),
$$

where

$$
\Delta\left(x_{1}^{a}, \ldots, x_{n}^{a}\right)=\prod_{1 \leq i<j \leq n}\left(x_{j}^{a}-x_{i}^{a}\right)
$$

Proof. Since we can find prime ideals $\left\{\mathbf{p}_{1}, \ldots, \mathbf{p}_{s}\right\}$ in $\mathbf{R}$ such that $V(I)=\bigcup_{i=1}^{s} V\left(\mathbf{p}_{i}\right)$ it will be sufficient to prove that there exists, for every prime ideal $\mathbf{p}$ in $\mathbf{R}$ with $\mathbf{p} \supset I$, an integer $a \geq 1$ with $V\left(\Delta\left(x_{1}^{a}, \ldots, x_{n}^{a}\right)\right) \supset V(\mathbf{p})$.

Indeed, let $I \subset \mathbf{p} \subset \mathbf{R}$ be a prime ideal. If $V(\mathbf{p})$ is finite, our assertion follows from Theorem 2.1. Assume therefore that $V(\mathbf{p})$ is infinite and set $R^{\prime}=\mathbf{R} / \mathbf{p}$. For every polynomial $f \in \mathbf{R}$ we denote by $f=f+\mathbf{p}$ the corresponding element in $R^{\prime}$ and we put $y_{i}=x_{i}^{\prime}, i=1, \ldots, n$. The Noether normalization lemma [AM] allows us to find an integer $1 \leq m \leq n$ and linear functions $z_{1}, \ldots, z_{m}$ of the elements $y_{1}, \ldots, y_{n}$ in $R^{\prime}$ such that the $z_{1}, \ldots, z_{m}$ are algebraically independent and each 
$y_{i}$ is algebraic and integral over $\mathbb{Q}\left[z_{1}, \ldots, z_{m}\right] \subset R^{\prime}$. Choose and fix monic polynomials $Q_{i} \in \mathbb{Q}\left[z_{1}, \ldots, z_{m}\right][y]$ such that $Q_{i}\left(y_{i}\right)=0$ for $i=1, \ldots, n$. We regard each $Q_{i}$ either as a polynomial in $y$ with coefficients in $\mathbb{Q}\left[z_{1}, \ldots, z_{m}\right]$ or as an element of $\mathbb{Q}\left[z_{1}, \ldots, z_{m}, y\right]$, and (ab)use the notation $Q_{i}=Q_{i}(y)=Q_{i}\left(z_{1}, \ldots, z_{m}, y\right), i=1, \ldots, n$, depending on our point of view. Let $\eta: V(\mathbf{p}) \rightarrow\left(\mathbb{Q}^{-}\right)^{m}$ be the surjective map

$$
\eta\left(\alpha_{1}, \ldots, \alpha_{n}\right)=\left(z_{1}\left(\alpha_{1}, \ldots, \alpha_{n}\right), \ldots, z_{m}\left(\alpha_{1}, \ldots, \alpha_{n}\right)\right),
$$

$\left(\alpha_{1}, \ldots, \alpha_{n}\right) \in V(\mathbf{p}) \subset\left(\mathbb{Q}^{-}\right)^{n}$. Then $\eta^{-1}\left(\beta_{1}, \ldots, \beta_{m}\right)$ is finite for every $\left(\beta_{1}, \ldots, \beta_{m}\right) \in\left(\mathbb{Q}^{-}\right)^{m}$, and $Q_{i}\left(\beta_{1}, \ldots, \beta_{m}, \alpha_{i}\right)=0$ for every $\left(\alpha_{1}, \ldots, \alpha_{n}\right) \in \eta^{-1}\left(\beta_{1}, \ldots, \beta_{m}\right)$ and $i=1, \ldots, n$. Put $K=\{u+v \sqrt{-1}$ : $u, v \in \mathbb{Q}\} \subset \mathbb{C}$. We denote by $\left\{P_{s}\left(z_{1}, \ldots, z_{m}\right): 0 \leq s \leq t\right\}$ the set of coefficients of the polynomials $Q_{i} \in \mathbb{Q}\left[z_{1}, \ldots, z_{m}\right][y], i=1, \ldots, n$, and claim that there exists a valuation $w$ of $K$ such that

$\Sigma=\left\{\left(\beta_{1}, \ldots, \beta_{m}\right) \in K^{m}:\left|P_{s}\left(\beta_{1}, \ldots, \beta_{m}\right)\right|_{w}=1\right.$ for every $\left.s=0, \ldots, t\right\}$

is dense in $\mathbb{C}^{m}$. Indeed, choose $w$ so that, for every $s=0, \ldots, t$, the coefficients $c_{i, s}$ of the polynomial $P_{s} \in \mathbb{Q}\left[z_{1}, \ldots, z_{m}\right]$ all satisfy $\left|c_{i, s}\right|_{w}=1$, and such that $\left|P_{s}(1, \ldots, 1)\right|_{w}=1$. If $r_{w}=\left\{\beta \in K:|\beta|_{w}<\right.$ $1\}$, then $q_{w}=1+r_{w} \subset K$ is dense in $\mathbb{C}$, and $\left|P_{s}\left(\beta_{1}, \ldots, \beta_{m}\right)\right|_{w}=1$ for all $\left(\beta_{1}, \ldots, \beta_{m}\right) \in q_{w}^{m}$ and $s=0, \ldots, t$. Hence $q_{w}^{m} \subset \Sigma$, and $\Sigma$ is dense in $\mathbb{C}^{m}$.

There exists an integer $d \geq 1$ such that, for every $\left(\beta_{1}, \ldots, \beta_{m}\right) \in$ $K^{m}$ and $\left(\alpha_{1}, \ldots, \alpha_{n}\right) \in \eta^{-1}\left(\beta_{1}, \ldots, \beta_{m}\right)$, the algebraic number field $K\left(\alpha_{1}, \ldots, \alpha_{n}\right)$ generated by $K$ and $\left(\alpha_{1}, \ldots, \alpha_{n}\right)$ has degree $\left(K\left(\alpha_{1}, \ldots, \alpha_{n}\right): \mathbb{Q}\right) \leq d$. Now consider elements $\left(\beta_{1}, \ldots, \beta_{m}\right) \in \Sigma$ and $\left(\alpha_{1}, \ldots, \alpha_{n}\right) \in \eta^{-1}\left(\beta_{1}, \ldots, \beta_{m}\right) \subset V(I)$. If $v$ is a valuation of $K\left(\alpha_{1}, \ldots, \alpha_{n}\right)$ above $w$, then $\left|\alpha_{i}\right|_{v}=1$ for $i=1, \ldots, n$, and there exist constants $c_{1}, \ldots, c_{n}$ in $K\left(\alpha_{1}, \ldots, \alpha_{n}\right)$ such that $c_{i} \neq 0$ for some $i \in\{1, \ldots, n\}$ and

$$
c_{1} \alpha_{1}^{m_{j}}+c_{2} \alpha_{2}^{m_{j}}+\cdots+c_{q} \alpha_{q}^{m_{j}}=0
$$

for every $j \geq 1$. After renumbering the $\alpha_{i}$ we may assume that $c_{1} \neq$ $0, \ldots, c_{q} \neq 0$, and $c_{q+1}=\cdots=c_{n}=0$. We denote by $\pi$ the rational prime below $v$ (or $w$ ), apply Theorem 2.1 to find an integer $a^{\prime}$ such that $1 \leq a^{\prime} \leq \pi^{2 d^{2}}$ and $\alpha_{i}^{a^{\prime}}=\alpha_{i^{\prime}}^{a^{\prime}}$ for some $1 \leq i<i^{\prime} \leq q$, and conclude that $\left(\alpha_{1}, \ldots, \alpha_{n}\right) \in V\left(\Delta\left(x_{1}^{a^{\prime}}, \ldots, x_{n}^{a^{\prime}}\right)\right)$.

We have shown that $\left(\alpha_{1}, \ldots, \alpha_{n}\right) \in V\left(\Delta\left(x_{1}^{a}, \ldots, x_{n}^{a}\right)\right)$ for all $\left(\alpha_{1}, \ldots, \alpha_{n}\right) \in \eta^{-1}(\Sigma)$, where $a=\pi^{2 d^{2}} !$ and $\Sigma \subset K^{m} \subset \mathbb{C}^{m}$ is dense in 
$\mathbb{C}^{m}$. Hence $V\left(\Delta\left(x_{1}^{a}, \ldots, x_{n}^{a}\right)\right) \cap V(\mathbf{p})$ is both Zariski-dense and closed in the Zariski-closed set $V(\mathbf{p})$, i.e. $V\left(\Delta\left(x_{1}^{a}, \ldots, x_{n}^{a}\right)\right) \supset V(\mathbf{p})$. Since $I \subset \mathbf{p} \subset \mathbf{R}$ was an arbitrary prime ideal we obtain (2.10).

2.3. Corollary. Let $K$ be a field of characteristic $0, q \geq 2$, and let $\alpha_{1}, \ldots, \alpha_{q}$ be elements in $K^{\times}$. Suppose that there exist an infinite sequence $m_{1}<m_{2}<\cdots$ in $\mathbb{N}$ and $c_{1}, \ldots, c_{q}$ in $K^{\times}$such that

$$
c_{1} \alpha_{1}^{m_{\jmath}}+c_{2} \alpha_{2}^{m_{\jmath}}+\cdots+c_{q} \alpha_{q}^{m_{\jmath}}=0
$$

for every $j \geq 1$. Then there exist integers $a, b$ such that $a \geq 1,0 \leq b<$ a,

$$
c_{1} \alpha_{1}^{j a+b}+c_{2} \alpha_{2}^{j a+b}+\cdots+c_{q} \alpha_{q}^{j a+b}=0 \text { for every } j \geq 0,
$$

and

$$
\alpha_{i}^{a}=\alpha_{i^{\prime}}^{a} \text { for some } i, i^{\prime} \text { with } 1 \leq i<i^{\prime} \leq q
$$

3. Mixing shapes and multiple mixing for group automorphisms. Let $(X, \mu)$ be a Lebesgue probability space, $d \geq 1$, and let $\alpha:(\mathbf{n}, x) \rightarrow$ $\alpha_{\mathbf{n}}(x), \mathbf{n} \in \mathbb{Z}^{d}, x \in X$, be a measure preserving $\mathbb{Z}^{d}$-action on $X$. The action $\alpha$ is mixing of order $r$ (or r-mixing) if, for all measurable sets $B_{0}, B_{1}, \ldots, B_{r}$ in $X$,

$$
\begin{aligned}
& \lim _{\substack{\mathbf{n}_{i} \in \mathbf{Z}^{d} \text { and } \\
\text { for all } i, j=1, \ldots, r, i \neq j \\
\mathbf{n}_{i} \rightarrow \mathbf{n}_{i}-\mathbf{n}_{j} \rightarrow \infty}} \mu\left(B_{0} \cap \alpha_{\mathbf{n}_{1}}\left(B_{1}\right) \cap \cdots \cap \alpha_{\mathbf{n}_{r}}\left(B_{r}\right)\right) \\
& \quad=\mu\left(B_{0}\right) \cdot \mu\left(B_{1}\right) \cdots \mu\left(B_{r}\right) .
\end{aligned}
$$

For a general discussion of mixing (i.e. 1-mixing) group actions we refer to [Dy] or [S1]. In the study of commuting automorphisms of compact groups one is naturally led to another concept related to multiple mixing, that of mixing shapes.

3.1. Definition. A shape is a nonempty subset of $\mathbb{Z}^{d}$. A shape $S=\left\{\mathbf{n}_{0}, \mathbf{n}_{1}, \ldots, \mathbf{n}_{r}\right\} \subset \mathbb{Z}^{d}$ is mixing under $\alpha$ if, for all measurable sets $B_{0}, B_{1}, \ldots, B_{r}$ in $X$,

$$
\begin{aligned}
\lim _{k \rightarrow \infty} \mu\left(\alpha_{k \mathbf{n}_{0}}\left(B_{0}\right) \cap \alpha_{k \mathbf{n}_{1}}\left(B_{1}\right) \cap \cdots \cap\right. & \left.\alpha_{k \mathbf{n}_{r}}\left(B_{r}\right)\right) \\
& =\mu\left(B_{0}\right) \cdot \mu\left(B_{1}\right) \cdots \mu\left(B_{r}\right) .
\end{aligned}
$$

Clearly, if $\alpha$ is $r$-mixing, then every shape $S \subset \mathbb{Z}^{d}$ of cardinality $r+1$ is mixing. The converse is probably not true unless, of course, $r=1$. 
Now assume that $X$ is a compact group with Haar measure $\lambda$ and that $d \geq 1$. A homomorphism $\alpha: \mathbf{n} \rightarrow \alpha_{\mathbf{n}}$ from $\mathbb{Z}^{d}$ into $\operatorname{Aut}(X)$ will be called a $\mathbb{Z}^{d}$-action on $X$ by automorphisms. We say that $(X, \alpha)$ satisfies the descending chain condition if, whenever $X_{1} \supset X_{2} \supset \cdots \supset X_{n} \supset \cdots$ is a decreasing sequence of closed, $\alpha$-invariant subgroups of $X$, there exists an integer $N \geq 1$ such that $X_{n}=X_{N}$ for all $n \geq N$. The pair $(X, \alpha)$ is expansive if there exists an open neighbourhood $N(\mathbf{1})$ of the identity element $\mathbf{1} \in X$ such that

$$
\bigcap_{\mathbf{n} \in \mathbf{Z}^{d}} \alpha_{\mathbf{n}}(N(\mathbf{1}))=\{\mathbf{1}\}
$$

It is not difficult to verify that $X$ must be metrizable if $(X, \alpha)$ is either expansive or satisfies the descending chain condition. Furthermore expansiveness implies the descending chain condition (cf. [KS, Theorem 5.2]). Finally, if $X$ is connected and $(X, \alpha)$ satisfies the descending chain condition, then $X$ is abelian (cf. [L]).

In order to analyze such dynamical systems $(X, \alpha)$ in more detail we have to introduce some notation. Let $R_{d}=\mathbb{Z}\left[u_{1}^{ \pm 1}, \ldots, u_{d}^{ \pm 1}\right]$ and $\mathbf{R}_{d}=\mathbb{Q} \cdot R_{d}=\mathbb{Q}\left[u_{1}^{ \pm 1}, \ldots, u_{d}^{ \pm 1}\right]$ be the rings of Laurent polynomials with integral (resp. rational) coefficients in the commuting variables $u_{1}, \ldots, u_{d}$, and put $u^{\mathbf{n}}=u_{1}^{n_{1}} \cdots \cdot u_{d}^{n_{d}} \in R_{d}$ for every $\mathbf{n}=\left(n_{1}, \ldots, n_{d}\right) \in$ $\mathbb{Z}^{d}$. If $N$ is an $R_{d}$-module and $\mathbf{n} \in \mathbb{Z}^{d}$ we define an automorphism $\beta_{\mathbf{n}}=\beta_{\mathbf{n}}^{N}$ of the additive group $N$ by

$$
\beta_{\mathbf{n}}^{N}(a)=u^{\mathbf{n}} \cdot a \quad \text { for every } a \in N
$$

and obtain a dual automorphism

$$
\alpha_{\mathbf{n}}=\alpha_{\mathbf{n}}^{N}=\hat{\beta}_{\mathbf{n}}^{N} \in \operatorname{Aut}(\hat{N}),
$$

where $\hat{N}$ is the dual group of the additive group $N$.

3.2. Proposition. Let $X$ be a compact, metrizable, abelian group, and let $\alpha: \mathbb{Z}^{d} \rightarrow \operatorname{Aut}(X)$ be a $\mathbb{Z}^{d}$-action on $X$ by automorphisms. Then there exists an $R_{d}$-module $N$ such that $\hat{N}=X$ and $\alpha_{\mathbf{n}}^{N}$ for every $\mathbf{n} \in \mathbb{Z}^{d}$. Conversely, if $N$ is an $R_{d}$-module, $X^{N}=\hat{N}$, and $\left\{\alpha_{\mathbf{n}}^{N}: \mathbf{n} \in \mathbb{Z}^{d}\right\} \subset$ $\operatorname{Aut}\left(X^{N}\right)$ is defined by (3.4) and (3.5), then $\alpha^{N}: \mathbf{n} \rightarrow \alpha_{\mathbf{n}}^{N}$ is a $\mathbb{Z}^{d}$. action on $X^{N}$ by automorphisms, and $\left(X^{N}, \alpha^{N}\right)$ satisfies the descending chain condition if and only if $N$ is finitely generated. The submodules $N^{\prime} \subset N$ are in one-to-one correspondence with the closed, $\alpha^{N}$-invariant subgroups $X^{N^{\prime}}$ of $X^{N}$, and this correspondence is given by $X^{N^{\prime}}=N^{\prime \perp}$, where $N^{\prime \perp} \subset \hat{N}$ is the annihilator of $\hat{N}$. Finally, if $M$ is a second $R_{d}$-module, $X^{M}=\hat{M}$, and $\left\{\alpha_{\mathbf{n}}^{M}: \mathbf{n} \in \mathbb{Z}^{d}\right\} \subset \operatorname{Aut}(\hat{M})$ is defined by (3.4) 
and (3.5), then every continuous algebraic isomorphism $\psi: X^{M} \rightarrow X^{N}$ with the property that $\psi \alpha_{\mathbf{n}}^{M}=\chi_{\mathbf{n}}^{N} \psi$ for all $\mathbf{n} \in \mathbb{Z}^{d}$ induces a dual module isomorphism $\eta: N \rightarrow M$.

Proof. [KS, Theorems 11.8 and 5.2].

We shall always assume that $N$ is finitely generated. It turns out that many of the dynamical properties of $\left(X^{N}, \alpha^{N}\right)$ (like ergodicity, mixing, and expansiveness) can be described in terms of the associated prime ideals of the $R_{d}$-module $N$ (cf. [S2]). Recall that a prime ideal $p \subset R_{d}$ is associated with $N$ if and only if there exists an element $a \in N$ such that

$$
p=\operatorname{ann}(a)=\left\{f \in R_{d}: f \cdot a=0\right\} .
$$

The set $\left\{p_{1}, \ldots, p_{m}\right\}$ of associated primes of $N$ is finite, and

$$
\bigcup_{i=1}^{m} p_{i}=\left\{f \in R_{d}: f \cdot a=0 \text { for some } a \neq 0 \text { in } N\right\} \text {. }
$$

For background we refer to [La].

We shall study higher order mixing properties of $\mathbb{Z}^{d}$-actions $\alpha: \mathbf{n} \rightarrow$ $\alpha_{\mathbf{n}}$ by automorphisms of a compact group $X$ under the assumption that $(X, \alpha)$ satisfies the descending chain condition. One checks easily that $\alpha$ is mixing (with respect to the Haar measure $\lambda$ ) if and only if $\alpha_{\mathbf{n}}$ is ergodic for every nonzero $\mathbf{n} \in \mathbb{Z}^{d}$ (cf. e.g. [KS, Theorem 2.4]). If $d=1, \alpha \in \operatorname{Aut}(X)$, and $\alpha$ is ergodic, then it is well known (and easy to see) that $n \rightarrow \alpha^{n}$ is $r$-mixing for every $r \geq 1$. As F. Ledrappier pointed out in [Le], the analogous result does not always hold if $d>1$ : there are closed, shift invariant subgroups $X \subset(\mathbb{Z} / 2 \mathbb{Z})^{Z^{2}}$ on which the shift-action of $\mathbb{Z}^{2}$ is mixing, but which have nonmixing shapes. In this section we prove the following.

3.3. Theorem. Let $X$ be a compact, abelian group, $d>1$, and let $\alpha: \mathbf{n} \rightarrow \alpha_{\mathbf{n}}$ be a mixing $\mathbb{Z}^{d}$-action on $X$ by automorphisms such that $(X, \alpha)$ satisfies the descending chain condition.

(1) If $X$ is connected, every shape $S \subset \mathbb{Z}^{d}$ is mixing.

(2) If $X$ is zero dimensional, the following conditions are equivalent:

(i) every shape $S \subset \mathbb{Z}^{d}$ is mixing;

(ii) the $\mathbb{Z}^{d}$-action $\alpha$ is $r$-mixing for every $r \geq 1$;

(iii) the set $\left\{p_{1}, \ldots, p_{m}\right\}$ of associated prime ideals of the $R_{d}$-module $M=\hat{X}$ is given by $p_{i}=\pi_{i} R_{d}, i=1, \ldots, m$, where $\left\{\pi_{1}, \ldots, \pi_{m}\right\}$ $\subset \mathbb{Z}$ are distinct rational primes. 
3.4. Corollary. Let $X$ be a compact, abelian group and let $\alpha: \mathbf{n} \rightarrow$ $\alpha_{\mathbf{n}}$ be a mixing $\mathbb{Z}^{d}$-action on $X$ by automorphisms such that $(X, \alpha)$ satisfies the descending chain condition. Let $M=\hat{X}$ be the finitely generated $R_{d}$-module arising from Proposition 3.2, and let $\left\{p_{1}, \ldots, p_{m}\right\}$ be the set of associated prime ideals of $M$. Then the following conditions are equivalent.

(1) Every shape $S \subset \mathbb{Z}^{d}$ is mixing under $\alpha$;

(2) for all $i$, either $p_{i} \cap \mathbb{Z}=\{0\}$, or $p_{i}=\pi_{i} R_{d}$ for a rational prime $\pi_{i} \in \mathbb{Z}$.

For the proofs of Theorem 3.3 and Corollary 3.4 we require several lemmas.

3.5. LemMA. Let $X$ be a compact, metrizable, abelian group with Haar measure $\lambda$ and dual group $\hat{X}$, and let $\alpha: \mathbb{Z}^{d} \rightarrow \operatorname{Aut}(X)$ be given as in the statement of Theorem 3.3. A shape $S=\left\{\mathbf{n}_{0}, \mathbf{n}_{1}, \ldots, \mathbf{n}_{r}\right\} \subset \mathbb{Z}^{d}$ is mixing under $\alpha$ if and only if, for all characters $\chi_{0}, \ldots, \chi_{r}$ in $\hat{X}$ with $\chi_{j} \neq 1$ for some $j \in\{0, \ldots, r\}$,

$$
\lim _{k \rightarrow \infty} \int\left(\chi_{0} \cdot \alpha_{k \mathbf{n}_{0}}\right) \cdot \cdots \cdot\left(\chi_{r} \cdot \alpha_{k \mathbf{n}_{r}}\right) d \lambda=0 .
$$

The action $\alpha$ is $r$-mixing if and only if, for all characters $\chi_{0}, \ldots, \chi_{r}$ in $\hat{X}$ with $\chi_{j} \neq 1$ for some $j \in\{0, \ldots, r\}$,

$$
\lim _{\substack{\mathbf{n}_{i} \in \mathbf{Z}^{d} \text { and } \mathbf{n}_{l} \rightarrow \infty, \mathbf{n}_{i}-\mathbf{n}_{j} \rightarrow \infty \\ \text { for all } i, j=1, \ldots, r, i \neq j}} \int\left(\chi_{0} \cdot \alpha_{\mathbf{n}_{0}}\right) \cdots \cdot\left(\chi_{r} \cdot \alpha_{\mathbf{n}_{r}}\right) d \lambda=0 .
$$

Proof. Expand the indicator functions of $B_{0}, \ldots, B_{r}$ in (3.2) as Fourier series.

3.6. LEMMA. If the group $X$ in Theorem 3.3 is connected, every shape $S \subset \mathbb{Z}^{d}$ is mixing.

Proof. We apply Lemma 3.2 to obtain a finitely generated $R_{d^{-}}$ module $M$ such that $X=\hat{M}$ and $\alpha_{\mathbf{n}}=\alpha_{\mathbf{n}}^{M}$ for every $\mathbf{n} \in \mathbb{Z}^{d}$. Let $\left\{p_{1}, \ldots, p_{m}\right\}$ be the set of prime ideals in $R_{d}$ associated with $M$ and let $\left\{W_{1}, \ldots, W_{m}\right\}$ be a reduced primary decomposition of 0 in $M$ such that $M / W_{i}$ is associated with $p_{i}$ for all $i=1, \ldots, m$ (cf. [La]). In particular,

$$
\bigcap_{i=1, \ldots, m} W_{i}=\{0\}
$$


Now assume that the $\mathbb{Z}^{d}$-action $\alpha$ is mixing, but that there exists a shape $S=\left\{\mathbf{n}_{0}, \ldots, \mathbf{n}_{r}\right\} \subset \mathbb{Z}^{d}$ which is not mixing. Lemma 3.5 implies that there exist elements $a_{i} \in M, i=0, \ldots, r$, and a sequence $k_{1}<$ $k_{2}<\ldots$ in $\mathbb{N}$ such that $a_{i} \neq 0$ for some $i \in\{0, \ldots, r\}$, but

$$
\left(\chi_{a_{0}} \cdot \alpha_{k_{j} \mathbf{n}_{0}}\right) \cdots \cdots\left(\chi_{a_{r}} \cdot \alpha_{k_{j} \mathbf{n}_{r}}\right)=1
$$

for every $j \geq 1$, where $\chi_{a} \in \hat{X}$ is the character of $X$ corresponding to an element $a \in M \cong \hat{X}$. According to (3.4) and (3.5), equation (3.10) is equivalent to the assertion that

$$
u^{k, \mathbf{n}_{0}} a_{0}+\cdots+u^{k_{j} \mathbf{n}_{r}} a_{r}=0 \text { for every } j \geq 1 \text {. }
$$

Equation (3.9) shows that there exists an integer $i \in\{1, \ldots, m\}$ such that $a_{j} \notin W_{i}$ for at least one $j \in\{0, \ldots, r\}$, and we set $N=M / W_{i}$, denote the image of $a_{i}$ in $N$ under the quotient map again by $a_{i}$, and regard (3.11) as an equation in $N$. Since the group $X$ is connected, the module $M$ is torsion-free as an additive group, and (3.6) shows that $p_{i} \cap \mathbb{Z}=\{0\}$ where $\mathbb{Z} \subset R_{d}$ is the set of constants. Since $N$ is associated with the prime ideal $p_{i}$ we know that the additive group $N$ is again torsion-free. Put $\mathbf{N}=\mathbb{Q} \otimes_{\mathbf{Z}} N$, and note that the natural homomorphism $l: N \rightarrow \mathbf{N}$ is injective, and that $\mathbf{N}$ is an $\mathbf{R}_{d}$-module with associated prime ideal $\mathbf{p}=\mathbb{Q} \otimes_{\mathbf{Z}} p_{i}$. An elementary argument allows us to choose sub- $\mathbf{R}_{d}$-modules $\mathbf{N}=\mathbf{N}_{0} \supset \cdots \supset \mathbf{N}_{s}=\{0\}$ of $\mathbf{N}$ such that, for every $j=0, \ldots, s-1, \mathbf{N}_{j} / \mathbf{N}_{j+1} \cong \mathbf{R}_{d} / \mathbf{q}_{j}$ for some prime ideal $\mathbf{p} \subset \mathbf{q}_{j} \subset \mathbf{R}_{d}$, and $\mathbf{q}_{s-1}=\mathbf{p}$. We set $a_{1, i}=\imath\left(a_{i}\right) \in \mathbf{N}$ and obtain that $a_{1, i} \neq 0$ for some $i \in\{1, \ldots, r\}$, but that

$$
u^{k_{j} \mathbf{n}_{0}} a_{1,0}+\cdots+u^{k_{j} \mathbf{n}_{r}} a_{1, r}=0 \text { for every } j \geq 1 .
$$

There exists an integer $t \in\{0, \ldots, s-1\}$ such that $\left\{a_{1,0}, \ldots, a_{1, r}\right\} \subset$ $\mathbf{N}_{t}$ and

$$
u^{k_{j} \mathbf{n}_{0}} a_{1,0}+\cdots+u^{k_{j} \mathbf{n}_{r}} a_{1, r}=0 \in \mathbf{N}_{t+1},
$$

but $a_{1, i} \notin \mathbf{N}_{t+1}$ for some $i \in\{0, \ldots, r\}$. After renumbering the $\mathbf{n}_{i}$ and $a_{1, i}$, if necessary, we obtain nonzero elements $b_{0}, \ldots, b_{r^{\prime}}$ in $\mathbf{R}_{d} / \mathbf{q}_{t}$, $0 \leq r^{\prime} \leq r$, with

$$
u^{k, \mathbf{n}_{0}} b_{0}+\cdots+u^{k, \mathbf{n}_{r^{\prime}}} b_{r^{\prime}}=0 \in \mathbf{R}_{d} / \mathbf{q}_{t}
$$

for every $j \geq 1$. Since $\mathbf{R}_{d} / \mathbf{q}_{t}$ is an integral domain we can apply Corollary 2.3 to the field of fractions of $\mathbf{R}_{d} / \mathbf{q}_{t}$ and conclude from (3.12) that there exist integers $b \geq 1$ and $i, i^{\prime} \in\left\{0, \ldots, r^{\prime}\right\}$ such that 
$i \neq i^{\prime}$ and $u^{b \mathbf{n}_{\imath}}-u^{b \mathbf{n}_{t^{\prime}}} \in \mathbf{q}_{t}$. If $u^{b \mathbf{n}_{t}}-u^{b \mathbf{n}_{\iota^{\prime}}} \notin \mathbf{p}$ we set

$$
a_{2, j}=\left(u^{b \mathbf{n}_{i}}-u^{b \mathbf{n}_{\iota^{\prime}}}\right) \cdot a_{1, j} \in \mathbf{N}_{t+1}, \quad j=0, \ldots, r .
$$

Then $a_{2, j} \neq 0$ for at least one $j \in\{1, \ldots, r\}$, but

$$
u^{k, \mathbf{n}_{0}} a_{2,0}+\cdots+u^{k, \mathbf{n}_{r}} a_{2, r}=0
$$

for every $j \geq 1$, and a repetition of the previous argument yields integers $c \geq 1$ and $j, j^{\prime} \in\{0, \ldots, r\}$ such that $j \neq j^{\prime}$ and $u^{c \mathbf{n}_{j}}-u^{c \mathbf{n}_{j}} \in$ $\mathbf{q}_{t+1}$. If $u^{c \mathbf{n}_{J}}-u^{c \mathbf{n}_{J^{\prime}}} \notin \mathbf{p}$ we continue this process. Since $\mathbf{q}_{s-1}=\mathbf{p}$ we eventually obtain integers $k \geq 1$ and $l, l^{\prime}$ with $l \neq l^{\prime}$ and $u^{k \mathbf{n}_{l}}-u^{k \mathbf{n}_{i^{\prime}}} \in \mathbf{p}$. Hence $u^{k \mathbf{n}_{l}}-u^{k \mathbf{n}_{l^{\prime}}} \in p_{i}$, and [S2, Theorem 3.5] shows that $\alpha$ is not mixing, in contradiction to our assumption. It follows that every shape $S \subset \mathbb{Z}^{d}$ must be mixing.

3.7. LemmA. Suppose that $X$ is zero dimensional, and that every shape $S \subset \mathbb{Z}^{d}$ is mixing under the $\mathbb{Z}^{d}$-action $\alpha$. If $\left\{p_{1}, \ldots, p_{m}\right\}$ is the set of associated primes of the $R_{d}$-module $M=\hat{X}$, then there exist rational primes $\pi_{1}, \ldots, \pi_{m}$ such that $p_{i}=\pi_{i} R_{d}$ for $i=1, \ldots, m$.

Proof. As in the proof of Lemma 3.6 we choose a reduced primary decomposition $\left\{M_{1}, \ldots, M_{m}\right\}$ of 0 in $M$ such that $M / M_{i}$ is associated with $p_{i}$ for every $i$. If $i \in\{1, \ldots, m\}$ is fixed, a standard argument allows us to find an element $a \in M$ such that $\operatorname{ann}(a)=\left\{f \in R_{d}: f \cdot a=\right.$ $0\}=p_{i}$, so that the submodule $N=R_{d} a \subset M$ is isomorphic to $R_{d} / p_{i}$. Since $N$ is a torsion group, there exists a rational prime (constant) $\pi_{i} \in p_{i}$. Suppose that $p_{i} \supsetneq \pi_{i} R_{d}$, fix a polynomial $f \in p_{i} \backslash \pi_{i} R_{d}$, and write $f=\sum_{\mathbf{n} \in S} c_{\mathbf{n}} \cdot u^{\mathbf{n}}$, where $S \subset \mathbb{Z}^{d}$ is a nonempty finite set and $c_{\mathbf{n}} \in\left\{1, \ldots, \pi_{i}-1\right\}$ for all $\mathbf{n} \in S$. For all $j \geq 1$,

$$
f^{\pi^{j}} \cdot a=\sum_{\mathbf{n} \in S} c_{\mathbf{n}} \cdot u^{\pi^{\prime} \mathbf{n}} \cdot a=0
$$

and

$$
\prod_{\mathbf{n} \in S}\left(\chi_{c_{\mathbf{n}} \cdot a} \cdot \alpha_{\pi / \mathbf{n}}\right)=1
$$

where, for every $\mathbf{n} \in S, \chi_{c_{\mathrm{n}} \cdot a}$ is the nontrivial character of $X$ corresponding to the nonzero element $c_{\mathrm{n}} \cdot a \in N \subset M$. By lemma 3.5 the set $S$ is not mixing, contrary to our assumption. We conclude that $p_{i}=\pi_{i} R_{d}$ for every $i=1, \ldots, m$. 
3.8. Lemma. Let $\left\{p_{1}, \ldots, p_{m}\right\}$ be the set of associated primes of the $R_{d}$-module $M=\hat{X}$, and assume that there exist rational primes $\pi_{1}, \ldots, \pi_{m}$ such that $p_{i}=\pi_{i} R_{d}$ for $i=1, \ldots, m$. Then $\alpha$ is $r$-mixing for every $r \geq 1$.

Proof. We use the notation of Lemma 3.7. Since $\left\{M_{1}, \ldots, M_{m}\right\}$ is a reduced primary decomposition of 0 in $M=\hat{X}, \bigcap_{i=1, \ldots, m} M_{i}=\{0\}$, the homomorphism $\eta: a \rightarrow\left(a+M_{1}, \ldots, a+M_{m}\right)$ from $M$ into $N_{1} \oplus$ $\cdots \oplus N_{m}$ is injective, where $N_{i}=M / M_{i}$, and the dual homomorphism $\tau: \hat{N}_{1} \oplus \cdots \oplus \hat{N}_{m} \rightarrow X$ is surjective. We define $\beta_{\mathbf{n}}^{N_{t}}$ and $\alpha_{\mathbf{n}}^{N_{t}}$ by (3.4) and (3.5), write $\beta_{\mathbf{n}}$ for the automorphism of $M$ dual to $\alpha_{\mathbf{n}}$, and note that

$$
\eta \cdot \beta_{\mathbf{n}}=\left(\beta_{\mathbf{n}}^{N_{1}} \oplus \cdots \oplus \beta_{\mathbf{n}}^{N_{m}}\right) \cdot \eta
$$

and

$$
\tau \cdot \alpha_{\mathbf{n}}=\left(\alpha_{\mathbf{n}}^{N_{1}} \oplus \cdots \oplus \alpha_{\mathbf{n}}^{N_{m}}\right) \cdot \tau
$$

for every $\mathbf{n} \in \mathbb{Z}^{d}$. We set $X_{i}=\hat{N}_{i}, i=1, \ldots, m$, and claim that the $\mathbb{Z}^{d}$-action $\mathbf{n} \rightarrow \alpha_{\mathbf{n}}^{N_{1}} \oplus \cdots \oplus \alpha_{\mathbf{n}}^{N_{m}}$ on $X_{1} \oplus \cdots \oplus X_{m}$ is $r$-mixing for every $r \geq 1$. In view of (3.14) this will prove that $\alpha$ is $r$-mixing for every $r \geq 1$.

Suppose that there exists an integer $r \geq 1$ such that the $\mathbb{Z}^{d}$-action $\mathbf{n} \rightarrow \alpha_{\mathbf{n}}^{N_{1}} \oplus \cdots \oplus \alpha_{\mathbf{n}}^{N_{m}}=\delta_{\mathbf{n}}$ on $X_{1} \oplus \cdots \oplus X_{m}$ is not $r$-mixing. Lemma 3.5 implies that there exist characters $\chi_{0}, \ldots, \chi_{r}$ of $X_{1} \oplus \cdots \oplus X_{m}$ such that $\chi_{i} \neq 1$ for some $i \in\{0, \ldots, r\}$, but

$$
\left(\chi_{0} \cdot \delta_{\mathbf{n}_{0, j}}\right) \cdot \cdots \cdot\left(\chi_{r} \cdot \delta_{\mathbf{n}_{r, j}}\right)=1
$$

for every $j \geq 1$, where $\left(\left(\mathbf{n}_{0, j}, \ldots, \mathbf{n}_{r, j}\right), j \geq 1\right)$ is a sequence in $\mathbb{Z}^{(r+1) d}$ with

$$
\lim _{j \rightarrow \infty} \mathbf{n}_{i, j}=\lim _{j \rightarrow \infty}\left(\mathbf{n}_{i, j}-\mathbf{n}_{i^{\prime}, j}\right)=\infty \quad \text { for all } i, i^{\prime} \in\{0, \ldots, r\} \text { with } i \neq i^{\prime} .
$$

There exists an integer $K \geq 1$ such that $\pi_{i}^{K} a=0$ for every $i \in$ $\{1, \ldots, m\}$ and every $a \in N_{i}$. Put $P_{i, j}=\left\{a \in N_{i}: \pi_{i}^{j} a=0\right\}$ and $P_{j}=P_{1, j} \oplus \cdots \oplus P_{m, j}, i=1, \ldots, m, j=0, \ldots, K$. We write $Y_{j}=P_{j}^{\perp}$ for the annihilator of $P_{j}$ in $X_{1} \oplus \cdots \oplus X_{m}$. Assume that, for some $j \geq 0$, the restriction of every $\chi_{i}$ to $Y_{j}$ is trivial. We shall complete the proof of this lemma by showing that the restriction of each $\chi_{i}$ to $Y_{j+1}$ is also trivial. An induction argument then shows that the characters $\chi_{0}, \ldots, \chi_{r}$ of $X_{1} \oplus \cdots \oplus X_{m}$ are all trivial, and the resulting contradiction implies that the $\mathbb{Z}^{d}$-actions $\mathbf{n} \rightarrow \delta_{\mathbf{n}}$ and $\mathbf{n} \rightarrow \alpha_{\mathbf{n}}$ are mixing of every order. 
For every $i=1, \ldots, m$, put $L_{i}=P_{i, j+1} / P_{i, j}$, and fix $i$ for the moment. Since $\pi_{i} L_{i}=\{0\}$ we may regard $L_{i}$ in an obvious manner as an $R_{\pi_{l}, d}$-module, where $R_{\pi_{i}, d}=\mathbb{Z}_{/ \pi_{i}}\left[u_{1}^{ \pm 1}, \ldots, u_{d}^{ \pm 1}\right]$ is the ring of Laurent polynomials in $u_{1}, \ldots, u_{d}$ with coefficients in the prime field $\mathbb{Z}_{/ \pi_{i}}=\mathbb{Z} / \pi_{i} \mathbb{Z}$. Put

$$
\mathbf{L}_{i}=k \otimes_{R_{\pi_{1}, d}} L_{i},
$$

where $k$ is the field of fractions of the integral domain $R_{\pi_{l}, d}$. Since $f \cdot a \neq 0$ for every $f \neq 0$ in $R_{\pi_{i}, d}$ and $a \neq 0$ in $L_{i}$, the natural injection $\imath_{i}: L_{i} \rightarrow \mathbf{L}_{i}$ is injective. Choose a basis $e_{1}, \ldots, e_{s}$ of the vector space $\mathbf{L}_{i}$ over $k$, assume without loss in generality that $\left\{e_{1}, \ldots, e_{s}\right\} \subset l_{i}\left(L_{i}\right)$, and find an element $\kappa \in k$ such that $l_{i}\left(L_{i}\right) \subset R_{\pi_{i}, d} \cdot \kappa e_{1}+\cdots+R_{\pi_{l}, d} \cdot \kappa e_{s}=$ $Q_{i} \subset \mathbf{L}_{i}$. In this manner we obtain an $R_{\pi_{i}, d}$-module $Q_{i} \cong\left(R_{\pi_{i}, d}\right)^{s}$ and an injective module homomorphism $l_{i}: L_{i} \rightarrow Q_{i}$. Since every $R_{\pi_{i}, d^{-}}$ module may also be regarded as an $R_{d}$-module we shall view $R_{\pi_{i}, d}$, $\left(R_{\pi_{i}, d}\right)^{S}$ and $Q_{i}$ as $R_{d}$-modules and $l_{i}$ as an injective $R_{d}$-module homomorphism. There is an obvious isomorphism $\psi: \hat{R}_{\pi_{l}, d} \rightarrow\left(\mathbb{Z}_{/ \pi_{l}}\right)^{\mathbb{Z}^{d}}$ such that

$$
\psi \alpha_{\mathbf{n}}^{R_{\pi_{l}, d}}=T_{\mathbf{n}} \quad \text { for every } \mathbf{n} \in \mathbb{Z}^{d},
$$

where $T_{\mathbf{n}}$ denotes the shift on $\left(\mathbb{Z}_{/ \pi_{t}}\right)^{\mathbb{Z}^{d}}$ defined by

$$
\left(T_{\mathbf{n}} \chi\right)(\mathbf{m})=\chi(\mathbf{m}+\mathbf{n}), \quad \mathbf{m} \in \mathbb{Z}^{d}
$$

This allows us to identify $\hat{Q}_{i}$ with $\left(\left(\mathbb{Z}_{/ \pi_{t}}\right)^{s}\right)^{\mathbf{Z}^{d}}$ and to assume that $\alpha_{\mathbf{n}}=$ $T_{\mathbf{n}}$ for all $\mathbf{n}$, where $T_{\mathbf{n}}$ now denotes the shift (3.16) on $\left(\left(\mathbb{Z}_{/} \pi_{i}\right)^{S}\right)^{\mathbb{Z}^{d}}$. The surjective homomorphism $\eta_{i}: \hat{Q}_{i} \rightarrow \hat{L}_{i}$ dual to $l_{i}: L_{i} \rightarrow Q_{i}$ satisfies that $\eta \alpha_{\mathbf{n}}^{Q_{l}}=\alpha_{\mathbf{n}}^{L_{1}} \eta$ for every $\mathbf{n} \in \mathbb{Z}^{d}$. Since the $\mathbb{Z}^{d}$-action $\mathbf{n} \rightarrow \alpha_{\mathbf{n}}^{Q_{1}} \oplus \cdots \oplus$ $\alpha_{\mathbf{n}}^{Q_{m}}$ on $\hat{Q}_{1} \oplus \cdots \oplus \hat{Q}_{m}$ is (isomorphic to) a cartesian product of full $d$ dimensional shifts it is $r$-mixing for every $r \geq 1$, and we conclude that the factor action $\mathbf{n} \rightarrow \alpha_{\mathbf{n}}^{L_{1}} \oplus \cdots \oplus \alpha_{\mathbf{n}}^{L_{m}}$ of $\mathbb{Z}^{d}$ on the group $\hat{L}_{1} \oplus \cdots \oplus \hat{L}_{m}$ is also mixing of every order. Note that $\hat{L}_{1} \oplus \cdots \oplus \hat{L}_{m}=Y_{j+1} / Y_{j}$, and that the $\mathbb{Z}^{d}$-action $\mathbf{n} \rightarrow \alpha_{\mathbf{n}}^{L_{1}} \oplus \cdots \oplus \alpha_{\mathbf{n}}^{L_{m}}$ is equal to the action on $Y_{j+1} / Y_{j}$ induced by $\mathbf{n} \rightarrow \delta_{\mathbf{n}}$. The characters $\chi_{0}, \ldots, \chi_{r}$ are trivial on $Y_{j}$ by assumption, and we may regard their restrictions to $Y_{j+1}$ as characters of $Y_{j+1} / Y_{j}$. However, since $\mathbf{n} \rightarrow \alpha_{\mathbf{n}}^{L_{1}} \oplus \cdots \oplus \alpha_{\mathbf{n}}^{L_{m}}$ is $r$ mixing, lemma 3.5 implies that these restrictions must be trivial, i.e. that $\chi_{i} \equiv 1$ on $Y_{j+1}$ for every $i \in\{0, \ldots, r\}$. As we have pointed out earlier, this completes the proof of the lemma. 
Proof of Theorem 3.3. Assertion (1) follows from Lemma 3.6. If $X$ is zero dimensional, then (i) $\Rightarrow$ (iii) by Lemma 3.7, and Lemma 3.8 shows that (iii) $\Rightarrow($ ii). The implication (ii) $\Rightarrow(\mathrm{i})$ is obvious. Theorem 3.3 is proved.

Proof of Corollary 3.4. Let $X^{0}$ be the connected component of the identity in $X$. If a shape $S=\left\{\mathbf{n}_{0}, \ldots, \mathbf{n}_{r}\right\} \subset \mathbb{Z}^{d}$ is not mixing under $\alpha$, choose characters $\chi_{0}, \ldots, \chi_{r}$ of $X$ such that at least one $\chi_{i}$ is nontrivial, but $\left(\chi_{0} \cdot \alpha_{k \mathbf{n}_{0}}\right) \cdot \ldots \cdot\left(\chi_{r} \cdot \alpha_{k \mathbf{n}_{r}}\right)=1$ for infinitely many $k \geq 1$ (cf. Lemma 3.5). From [S2, Corollary 3.6] we know that the restriction of $\alpha$ to the closed, $\alpha$-invariant subgroup $X^{0}$ of $X$ is still mixing, and Lemma 3.5 implies that the restrictions of the characters $\chi_{i}$ to $X^{0}$ must all be trivial. Hence the characters $\chi_{0}, \ldots, \chi_{r}$ can be regarded as elements of $\hat{Y}$, where $Y=X / X^{0}$. The set of associated prime ideals of the $R_{d}$-module $N=\hat{Y} \subset M$ (cf. Proposition 3.2) is given by $\left\{p_{i}: 1 \leq i \leq m\right.$ and $\left.p_{i} \cap \mathbb{Z} \neq \varnothing\right\}$, and lemma 3.8 implies that there exist an $i \in\{1, \ldots, m\}$ and a rational prime $\pi_{i}$ such that $p_{i} \supsetneq \pi_{i} R_{d}$. This shows that $(2) \Rightarrow(1)$.

If every shape $S \subset \mathbb{Z}^{d}$ is mixing, then every shape is also mixing for the quotient action induced by $\alpha$ on the zero dimensional group $Y=X / X^{0}$, and Lemma 3.7 implies that every prime ideal associated with the module $N=\hat{Y} \subset M$ is of the form $p_{i}=\pi_{i} R_{d}$ for some rational prime $\pi_{i}$. This proves that $(1) \Rightarrow(2)$.

3.9. Problem. Let $X$ be a compact, connected abelian group and let $\alpha: \mathbf{n} \rightarrow \alpha_{\mathbf{n}}$ be a mixing $\mathbb{Z}^{d}$-action on $X$ by automorphisms. Is $\alpha$ $r$-mixing for every $r \geq 1$ ?

\section{REFERENCES}

[AM] M. F. Atiyah and I. G. Macdonald, Introduction to Commutative Algebra, Addison-Wesley, 1969.

[Dy] H. A. Dye, On the ergodic mixing theorem, Trans. Amer. Math. Soc., 118 (1965), 123-130.

[KS] B. Kitchens and K. Schmidt, Automorphisms of compact groups, Ergodic Theory Dynamical Systems (to appear).

[L] P.-F. Lam, On expansive transformation groups, Trans. Amer. Math. Soc., 150 (1970), 131-138.

[La] S. Lang, Algebra, Addison-Wesley, 1984 (2nd edition).

[Le] F. Ledrappier, Un champ markovien peut être d'entropie nulle et mélangeant, C. R. Acad. Sci. Paris, Ser. A., 287 (1978), 561-562.

[Ma] K. Mahler, Eine arithmetische Eigenschaft der Taylor-koeffizienten rationaler Funktionen, Proc. Acad. Sci. Amsterdam, 38 (1935), 50-60. 
[S1] K. Schmidt, Asymptotic properties of unitary representations and mixing, Proc. London Math. Soc., 48 (1984), 445-460.

[S2] - Automorphisms of compact abelian groups and affine varieties, preprint.

Received April 15, 1988.

INSTITUTE FOR ADVANCED STUDY

PRINCETON, NJ 08540

AND

UNIVERSITY OF WARWICK

COVENTRY CV4 7AL, UK 



\section{PACIFIC JOURNAL OF MATHEMATICS EDITORS}

\author{
V. S. VARADARAJAN \\ (Managing Editor) \\ University of California \\ Los Angeles, CA 90024 \\ Herbert Clemens \\ University of Utah \\ Salt Lake City, UT 84112 \\ THOMAS ENRIGHT \\ University of California, San Diego \\ La Jolla, CA 92093
}

R. FINN

Stanford University

Stanford, CA 94305

HeRmann FlaschKa

University of Arizona

Tucson, AZ 85721

VAUGHAN F. R. Jones

University of California

Berkeley, CA 94720

STEVEN KeRCKHOFF

Stanford University

Stanford, CA 94305
ROBION KIRBY

University of California

Berkeley, CA 94720

C. C. MOORE

University of California

Berkeley, CA 94720

HAROLD STARK

University of California, San Diego

La Jolla, CA 92093

\section{ASSOCIATE EDITORS}
R. ARENS
E. F. BECKENBACH
B. H. NeUMANN
F. WolF
K. YoshidA (1906-1982)

\section{SUPPORTING INSTITUTIONS}

UNIVERSITY OF ARIZONA

UNIVERSITY OF BRITISH COLUMBIA

CALIFORNIA INSTITUTE OF TECHNOLOGY

UNIVERSITY OF CALIFORNIA

MONTANA STATE UNIVERSITY

UNIVERSITY OF NEVADA, RENO

NEW MEXICO STATE UNIVERSITY

OREGON STATE UNIVERSITY

\author{
UNIVERSITY OF OREGON \\ UNIVERSITY OF SOUTHERN CALIFORNIA \\ STANFORD UNIVERSITY \\ UNIVERSITY OF HAWAII \\ UNIVERSITY OF TOKYO \\ UNIVERSITY OF UTAH \\ WASHINGTON STATE UNIVERSITY \\ UNIVERSITY OF WASHINGTON
}

The Supporting Institutions listed above contribute to the cost of publication of this Journal, but they are not owners or publishers and have no responsibility for its content or policies.

Mathematical papers intended for publication in the Pacific Journal of Mathematics should be in typed form or offset-reproduced (not dittoed), double spaced with large margins. Please do not use built up fractions in the text of the manuscript. However, you may use them in the displayed equations. Underline Greek letters in red, German in green, and script in blue. The first paragraph must be capable of being used separately as a synopsis of the entire paper. In particular it should contain no bibliographic references. Please propose a heading for the odd numbered pages of less than 35 characters. Manuscripts, in triplicate, may be sent to any one of the editors. Please classify according to the scheme of Math. Reviews, Index to Vol. 39. Supply name and address of author to whom proofs should be sent. All other communications should be addressed to the managing editor, or Elaine Barth, University of California, Los Angeles, California 90024.

There are page-charges associated with articles appearing in the Pacific Journal of Mathematics. These charges are expected to be paid by the author's University, Government Agency or Company. If the author or authors do not have access to such Institutional support these charges are waived. Single authors will receive 50 free reprints; joint authors will receive a total of 100 free reprints. Additional copies may be obtained at cost in multiples of 50 .

The Pacific Journal of Mathematics is issued monthly as of January 1966. Regular subscription rate: $\$ 190.00$ a year (5 Vols., 10 issues). Special rate: $\$ 95.00$ a year to individual members of supporting institutions.

Subscriptions, orders for numbers issued in the last three calendar years, and changes of address should be sent to Pacific Journal of Mathematics, P.O. Box 969, Carmel Valley, CA 93924, U.S.A. Old back numbers obtainable from Kraus Periodicals Co., Route 100, Millwood, NY 10546.

The Pacific Journal of Mathematics at P.O. Box 969, Carmel Valley, CA 93924 (ISSN 0030-8730) publishes 5 volumes per year. Application to mail at Second-class postage rates is pending at Carmel Valley, California, and additional mailing offices. Postmaster: send address changes to Pacific Journal of Mathematics, P.O. Box 969, Carmel Valley, CA 93924.

\section{PUBLISHED BY PACIFIC JOURNAL OF MATHEMATICS, A NON-PROFIT CORPORATION}




\section{Pacific Journal of Mathematics}

Vol. 137, No. $2 \quad$ February, 1989

Alain Connes and E. J. Woods, Hyperfinite von Neumann algebras and Poisson boundaries of time dependent random walks ............ 225

R. Coquereaux and D. Kastler, Remarks on the differential envelopes of

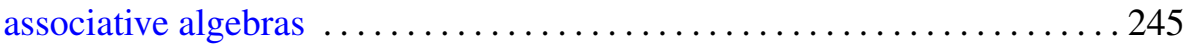

Uffe Haagerup, The injective factors of type $\mathrm{III}_{\lambda}, 0<\lambda<1 \ldots \ldots \ldots 265$

Vaughan Jones, On knot invariants related to some statistical mechanical

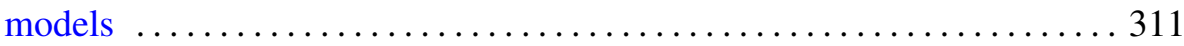

Paul Scott Muhly, Kichi-Suke Saito and Baruch Solel, Coordinates for triangular operator algebras. II ......................... 335

Klaus Schmidt, Mixing automorphisms of compact groups and a theorem by Kurt Mahler ........................................ 371

Irving E. Segal, Algebraic characterization of the vacuum for quantized

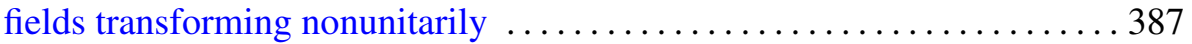

Colin Eric Sutherland and Masamichi Takesaki, Actions of discrete amenable groups on injective factors of type $\mathrm{III}_{\lambda}, \lambda \neq 1 \ldots \ldots \ldots \ldots 405$ 